\title{
PMU-Based System Identification for a Modified Classic Generator Model
}

\author{
Yasser Wehbe, Lingling Fan, Senior Member, IEEE
}

\begin{abstract}
The paper proposes to use PMU measurements (voltage phasor, real and reactive powers) for system identification. A second-order synchronous generator model with real and reactive power as input and voltage phasor as output will be developed in this paper based on the two-axis synchronous generator dynamic model. Parameters of the model will be identified based on the PMU measurements using least square estimation technique. Two case studies are presented: a synchronous generator estimation and a power subsystem estimation. In both cases, the system will be represented by the proposed model with parameters identified from PMU measurements. Time-domain simulation results will validate the accuracy of the proposed model and its identification. The contribution of this paper is twofold: i) A model suitable for PMU measurement based identification is developed; ii) better accuracy of system parameter estimation can be achieved using the proposed model compared to the classical model.
\end{abstract}

Index Terms-Least square estimation, parameter estimation, synchronous machine, system identification.

\section{INTRODUCTION}

Phasor Measurements Units (PMU) provide time stamped phasor data such as voltage magnitude $(V)$ and angle $(\theta)$, active power $\left(P_{e}\right)$ and reactive power $\left(Q_{e}\right)$, at a reporting rate of 20-60 Hz. Due to such characteristics, PMU is regarded as in important tool in Wide Area Measurement System (WAMS) [1]. The objective of this research is to use PMU data for system identification, i.e., given time series data of input and output of a system and its dynamic model structure, the entire set of the parameters of the model will be identified using least square estimation.

Due to the low sampling rate of PMU data, PMU measurements do not reflect electromagnetic dynamics adequately. However, the sampling rate is high enough to reflect the lowfrequency electromechanical dynamics in power grids. Compared to some of the approaches in the literature, where all kinds of measurements can be obtained, PMU measurements are limited to voltage phasors and powers. Therefore, the dynamic model suitable for PMU data application will focus on electromechanical dynamics. Meanwhile, the parameters identified will be a subset of generator parameters.

Two estimation approaches are dominant: Kalman Filter (KF) based estimation and least squares based estimation. KF approach is a Bayesian recursive method aiming at reducing a covariance matrix whereas the least squares approach uses a non Bayesian methods considering the complete data set in the time window. KF approaches have been proposed to

Y. Wehbe is with Ventyx, Texas. L. Fan is with the Department of Electrical Engineering, University of South Florida, Tampa, FL 33620, email: linglingfan@usf.edu. estimate synchronous machine electromechanical parameters using PMU measurements. In [2], extended Kalman filter (EKF) based method is proposed to calibrate the parameters of a classical generator. In [3], EKF is applied to calibrate the multi-machine power system parameters. Our research in [4] has also examined EKF application for PMU data based estimation and the Unscented Kalman Filter in [5].

Least squares based system identification has been seen plenty in the literature in variety of applications. The objective of system identification is to compare the output of a system with the projected output of the model given the same input. The parameters of the model will be optimized to reach a minimum error squares over the experimental period. System identification has been used in power electronic research in identifying a power converter model [6], [7], to model large signal power electronics systems [8], and to estimate DC link model parameters in VSC-HVDC system [9]. It has also been used in generator parameter identification [10], Prony analysis [11], [12], ARX-based generator model identification [13], [14] and in finding the state space system for multi-input multioutput models of power systems [15].

In PMU data based estimation, least square estimation based system identification approach has not been examined. This approach will be investigated in this paper.

A key challenge in least square estimation based system identification is the difficulty to estimate some parameters since they are insensitive to the output data. This makes the estimation of some parameters not reliable. Burth et al. [16] points out to the difficulty in estimating synchronous machine parameters based on measurements such as terminal voltage and current. This is especially a problem in PMU measurements since these measurements are the only measurements can be obtained and used.

Burth et al. [16] suggests to apply parameters sub-set selection to find a best set of parameters that can be estimated with reasonable amount of precision. In [16], a Hessian matrix of the objective function is applied to find the parameters subset. Another approach for sub-set selection is the study of sensitivity matrix as shown by Cintron-Arias et al. [17]. The two approaches are correlated and both are based on Jacobian calculation. In this research, sensitivity matrix will be used to demonstrate the choice of model order.

The rest of this paper is organized as follows: Section II will derive a linear model suitable for PMU data based system identification. Section III presents case studies applying Matlab System Identification Toolbox. A single machine identification and a an area identification are presented. Section IV presents the conclusion of this paper. 


\section{Proposed Model Suitable for PMU Data based IDENTIFICATION}

To facilitate system identification, PMU measurements will be separated into two sets: the input and the output. The active and reactive powers will be treated as the input and the voltage magnitude and phase will be considered as the output. The purpose of this section is to build a linearized

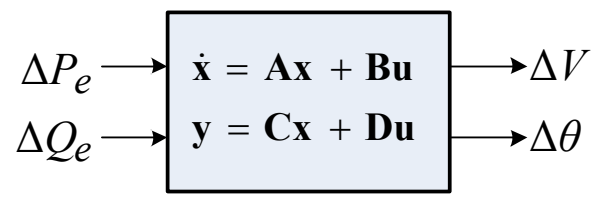

Fig. 1. Proposed state space model

state-space model as shown in Fig. 1. In order to identify which set of parameters can be better represented in the PMU measurements, sensitivity analysis will be conducted. Furthermore, a reduced-order model will be developed.

\section{A. Subset selection}

Synchronous machine two-axis model with no governor nor exciter controls and ignoring the sub-transient dynamics [18] can be described by:

$$
\begin{aligned}
\frac{\partial \delta^{\prime}}{\partial t} & =\omega-\omega_{0} \\
\frac{2 H}{\omega_{0}} \frac{\partial \omega}{\partial t} & =P_{m}-P_{e} \\
T_{d o}^{\prime} \frac{\partial E_{q}^{\prime}}{\partial t} & =-E_{q}^{\prime}-\left(x_{d}-x_{d}^{\prime}\right) I_{d}+E_{f d} \\
T_{q o}^{\prime} \frac{\partial E_{d}^{\prime}}{\partial t} & =-E_{d}^{\prime}-\left(x_{q}-x_{q}^{\prime}\right) I_{d}
\end{aligned}
$$

The internal voltage, current and terminal voltage relationship is expressed as follows.

$$
\left[E_{d}^{\prime}+\left(x_{d}^{\prime}-x_{q}^{\prime}\right) I_{q}+E_{q}^{\prime}\right]=j x_{d}^{\prime}\left(I_{d}+j I_{q}\right)+V e^{j\left(\theta-\delta^{\prime}+\pi / 2\right)}
$$

Phasor diagram in 2 also explains the above relationship.

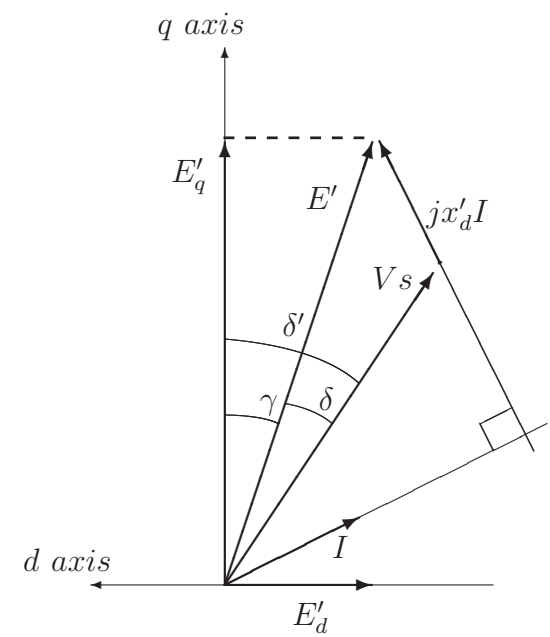

Fig. 2. Phasor diagram for a classical model from [19].
[17] proposes to use sensitivity matrix to select a subset of parameters to be estimated. Sensitivity matrix can be used study the impact of the various parameters on the output of a system, in other words, it tries to find the most influential and the least influential parameter on the output.

In this research, for a two-axis generator model, the machine parameter set is defined as $\mathbf{M}=\left\{H, x_{d}^{\prime}, x_{q}, x_{d}, T_{d o}^{\prime}, T_{q o}^{\prime}\right\}$ where $H$ is the inertia, $x_{d}^{\prime} \mathrm{d}$-axis transient reactance, $x_{d}$ and $x_{q}$ are the dq-axis reactances, $T_{d o}^{\prime}$ and $T_{q o}^{\prime}$ are the dq-axis opencircuit time constants. The sensitivity matrix of the system (20)-(21) is the Jacobian matrix $\chi$ of the output $\mathbf{Y}$ wrt. the parameter set $\mathbf{M}$.

$$
\chi_{i j}=\frac{\partial Y_{i}}{\partial M_{j}}
$$

where $i$ denotes $i$-th output and $j$ denotes $j$-th parameter. $Y_{i}$ is the $i^{\text {th }}$ measurement and has $N$ samples.

Accordingly, $\chi$ for the system will be $N \times 6$ where $N$ is the total number of samples.

The importance of the sensitivity matrix in least squares based estimations (like system identification) comes from the objective of the least squares estimation of minimizing the output error by manipulating $\mathbf{M}$ around a value $M_{0}$ [17] to find its estimate $\hat{M}$ :

$$
\hat{\mathbf{M}}=\underset{\mathbf{M}}{\arg \min } \sum_{i=1}^{N}(Y(i)-\hat{Y}(i \mid M))^{2}
$$

The estimated parameter set has an expression as follows:

$$
\hat{\mathbf{M}}=M_{0}+\left(\chi^{T} \chi\right)^{-1} \chi^{T} \zeta
$$

where $\zeta$ is the $1 \times N$ error (noise) matrix associated with the output equation. A good estimation of $\mathbf{M}$ will reduce the impact of $\zeta$ by having $\left(\chi^{T} \chi\right)$ far from singularity. Should $\left(\chi^{T} \chi\right)$ be close to singular, then $\left(\chi^{T} \chi\right)^{-1}$ will amplify the impact of $\zeta$ and distorts the estimation of $\mathbf{M}$.

Singular value decomposition (SVD) of $\chi$ provides an insightful relation between each parameter singular value and the possibility of the least squares estimation to find a good value of the parameter. The singular value decomposition of of $\chi$ is $\chi=\Upsilon \mathbf{S} \Omega^{T}$. where $\Upsilon$ is an $N \times N$ orthogonal matrix, $\Omega$ is a $6 \times 6$ orthogonal matrix, and $\mathbf{S}$ is the singular value matrix and is $\chi$ is $N \times 6$ matrix. The first 6 diagonal elements of $\mathbf{S}$ are the singular values of $\chi$ and the rest of the matrix equals to 0.

The estimation of $\mathbf{M}$ around $M_{0}$ can now be written as [17]:

$$
\hat{\mathbf{M}}=M_{0}+\sum_{i=1}^{6} \frac{o_{i} u_{i}^{T}}{s_{i}} \zeta
$$

where $o_{i}, u_{i}$ are the ith columns of $\Omega$ and $\Upsilon . s_{i}$ is the ith diagonal value of $\mathbf{S}$.

Equation (9) shows the inverse proportional impact of the singular value $s_{i}$ associated with a parameter $M_{i}$. As $s_{i}$ decreases the error $\zeta$ introduces more distortion on the estimated parameter and leads to a larger deviation from the correct value. Accordingly, it is better to estimate the parameters with high singular values. In order to find the singular values, the matrix $\chi$ needs to be calculated. 
$\chi$ can be calculated analytically [20] in case the the associated differential equations are simple. In other cases, $\chi$ can be calculated numerically. The sensitivity matrix $\chi$ was calculated numerically in [21] using finite differences by perturbing each parameter $M_{i}$ aside by a value $h$ and then recording the output of the system. The recorded output before $(Y)$ and after $\left(Y_{h}\right)$ perturbation are used for the Jacobian calculation: $\chi=\frac{Y_{h}-Y}{h}$. Following the calculation of the $\chi, \mathrm{SVD}$ is performed in order to extract $\mathbf{S}$.

$$
\mathbf{S}=\left[\begin{array}{cccccc}
3.9 & 0 & 0 & 0 & 0 & 0 \\
0 & 0.2 & 0 & 0 & 0 & 0 \\
0 & 0 & 0.12 & 0 & 0 & 0 \\
0 & 0 & 0 & 0.08 & 0 & 0 \\
0 & 0 & 0 & 0 & 0.04 & 0 \\
0 & 0 & 0 & 0 & 0 & 0.03
\end{array}\right]
$$

It was found that $x_{d}^{\prime}$ is associated with $3.9, H$ with $0.2, x_{q}$ with $0.12, x_{d}$ with $0.08, T_{q o}^{\prime}$ with $0.04, T_{D}^{\prime} d o$ with 0.03 . Therefore, it is obvious that $x_{d}^{\prime}$ and $H$ can be estimated reliably, while the two time constants cannot be estimated as reliably as $x_{d}^{\prime}$ and $H$. In the following subsection, a second-order generator model will be derived from the two-axis model. By reducing the order of the model, the two time constants no longer need to be estimated.

\section{B. Modified Classical Generator Model}

For the two-axis synchronous generator model, the powers can be expressed by terminal voltage and current or terminal voltage and internal voltages. Equations (11) and (12) present the powers:

$$
\begin{gathered}
\left\{\begin{array}{l}
P_{e}=I_{d} V \sin \left(\delta^{\prime}-\theta\right)+I_{q} V \cos \left(\delta^{\prime}-\theta\right) \\
Q_{e}=I_{d} V \cos \left(\delta^{\prime}-\theta\right)-I_{q} V \sin \left(\delta^{\prime}-\theta\right)
\end{array}\right. \\
\left\{\begin{array}{l}
P_{e}=\frac{E_{q}^{\prime} V \sin \left(\delta^{\prime}-\theta\right)-E_{d}^{\prime} V \cos \left(\delta^{\prime}-\theta\right)}{x_{d}^{\prime}} \\
Q_{e}=\frac{E_{q}^{\prime} V \cos \left(\delta^{\prime}-\theta\right)+E_{d}^{\prime} V \sin \left(\delta^{\prime}-\theta\right)-V^{2}}{x_{d}^{\prime}}
\end{array}\right.
\end{gathered}
$$

Note that (12) has an assumption of neglecting the transient saliency. Therefore $x_{d}^{\prime}=x_{q}^{\prime}$.

The synchronous machine classical model is obtained by reducing the two-axis model when setting $T_{q o}^{\prime}$ is set to zero (i.e. ignoring the quick $q$ axis dampers dynamics) and $T_{d o}^{\prime}$ is extended to $\infty$ therefore $E_{q}^{\prime}$ is a constant. Consequently, such simplification keeps the electromechanical dynamics in (1) and (2) and completely decouples from $E_{d}^{\prime}$ and $E_{q}^{\prime}$ dynamics. In classical model, the electric circuit, the generator is a voltage source $E^{\prime}$ behind a transient reactance $x_{d}^{\prime}$. The angle between $E^{\prime}$ and $V$ is $\delta$. The difference between the voltage source angle $\delta$ and the rotor angle $\delta^{\prime}$ is ignored in classical generator model.

In this paper, this difference will not be ignored. According to [19] and [18] the difference $\gamma$ between the rotor angle $\delta^{\prime}$ and the voltage source angle $\delta$ is almost constant and is negligible when studying angle dynamics. Fig. 2 [19] shows $\gamma, \delta^{\prime}$, and $\delta$. Accordingly:

$$
\delta^{\prime}=\delta+\gamma \Rightarrow \dot{\Delta \delta^{\prime}} \quad=\dot{\Delta} \delta+\dot{\Delta} \gamma
$$

Careful attention at $\gamma$ (Fig. 2) shows the following:

$$
\begin{aligned}
\gamma & =\tan ^{-1}\left(\frac{E_{d}^{\prime}}{E_{q}^{\prime}}\right) \Rightarrow \\
\dot{\Delta} \gamma & =-\frac{E_{d 0}^{\prime}}{E_{d 0}^{\prime 2}+E_{q 0}^{\prime 2}} \Delta \dot{E}_{q}^{\prime}+\frac{E_{q 0}^{\prime}}{E_{d 0}^{\prime 2}+E_{q 0}^{\prime 2}} \Delta \dot{E}_{d}^{\prime}
\end{aligned}
$$

When $E_{d}^{\prime}$ and $E_{q}^{\prime}$ dynamics are ignored $\dot{\Delta} \gamma=0$. In this paper, the effect of dynamics will be considered. Based on (13) and (14):

$$
\begin{aligned}
\dot{\Delta \delta} & =\dot{\Delta} \delta^{\prime}-\dot{\Delta \gamma} \\
\Rightarrow & \dot{\Delta} \delta=\Delta \omega+\frac{E_{d 0}^{\prime}}{E_{d 0}^{\prime 2}+E_{q 0}^{\prime 2}} \Delta \dot{E}_{q}^{\prime}-\frac{E_{q 0}^{\prime}}{E_{d 0}^{\prime 2}+E_{q 0}^{\prime 2}} \Delta \dot{E}_{d}^{\prime}
\end{aligned}
$$

We need to find $\left(\Delta \dot{E}_{q}^{\prime}\right)$ and $\left(\Delta \dot{E}_{q}^{\prime}\right)$. Based on the two-axis generator model, we have:

$$
\begin{aligned}
& \left(\Delta \dot{E}_{q}^{\prime}\right)=\frac{1}{T_{d o}^{\prime}}\left[-\Delta E_{q}^{\prime}-\left(x_{d}-x_{d}^{\prime}\right) \Delta I_{d}+\Delta E_{f d}\right] \\
& \left(\Delta \dot{E}_{d}^{\prime}\right)=\frac{1}{T_{q o}^{\prime}}\left[-\Delta E_{d}^{\prime}-\left(x_{d}-x_{d}^{\prime}\right) \Delta I_{q}\right.
\end{aligned}
$$

Since $\Delta P_{e}$ and $\Delta Q_{e}$ are treated as the input to the model, the derivatives $\left(\Delta \dot{E}_{q}^{\prime}\right),\left(\Delta \dot{E}_{d}^{\prime}\right)$ should be expressed in terms of states $\left(\Delta E_{q}^{\prime}, \Delta E_{d}^{\prime}\right)$ and powers $\left(\Delta P_{e}\right.$ and $\left.\Delta Q_{e}\right)$ not in terms of $\Delta I_{d}$ and $\Delta I_{q} . \Delta I_{d}$ and $\Delta I_{q}$ can be expressed in terms of $\Delta E_{d}^{\prime}, \Delta E_{q}^{\prime}, \Delta P_{e}$, and $\Delta Q_{e}$ using (11) and (12) (by removing $V \sin \left(\delta^{\prime}-\theta\right)$ and $\left.V \cos \left(\delta^{\prime}-\theta\right)\right)$. Accordingly, the small signal system $^{1}(16)$ and (17) will become:

$$
\begin{aligned}
{\left[\begin{array}{c}
\Delta \dot{E_{q}^{\prime}} \\
\Delta \dot{E_{d}^{\prime}}
\end{array}\right]=} & {\left[\begin{array}{ll}
J_{\dot{E}_{q}^{\prime} E_{q}^{\prime}} & J_{\dot{E}_{q}^{\prime} E_{d}^{\prime}} \\
J_{\dot{E}_{d}^{\prime} E_{q}^{\prime}} & J_{\dot{E}_{d}^{\prime} E_{d}^{\prime}}
\end{array}\right]\left[\begin{array}{l}
\Delta E_{q}^{\prime} \\
\Delta E_{d}^{\prime}
\end{array}\right]+} \\
& {\left[\begin{array}{cc}
J_{\dot{E}_{q}^{\prime} P_{e}} & J_{\dot{E}_{q}^{\prime} Q_{e}} \\
J_{\dot{E}_{d}^{\prime} P_{e}} & J_{\dot{E}_{d}^{\prime} Q_{e}}
\end{array}\right]\left[\begin{array}{c}
\Delta P_{e} \\
\Delta Q_{e}
\end{array}\right]+\left[\begin{array}{c}
1 / T_{d o}^{\prime} \\
0
\end{array}\right] \Delta E_{f d} }
\end{aligned}
$$

Based on the power expression in (12), the terminal voltage phase angle and magnitude can also be expressed in terms of states, and power:

$$
\begin{aligned}
{\left[\begin{array}{c}
\Delta \theta \\
\Delta V
\end{array}\right]=} & {\left[\begin{array}{l}
1 \\
0
\end{array}\right] \Delta \delta^{\prime}+\left[\begin{array}{ll}
J_{\theta P_{e}} & J_{\theta Q_{e}} \\
J_{V P} & J_{V Q_{e}}
\end{array}\right]\left[\begin{array}{c}
\Delta P_{e} \\
\Delta Q_{e}
\end{array}\right]+} \\
& {\left[\begin{array}{ll}
J_{\theta E_{q}^{\prime}} & J_{\theta E_{d}^{\prime}} \\
J_{V E_{q}^{\prime}} & J_{V E_{d}^{\prime}}
\end{array}\right]\left[\begin{array}{l}
\Delta E_{q}^{\prime} \\
\Delta E_{d}^{\prime}
\end{array}\right] }
\end{aligned}
$$

$\left(J_{\theta P_{e}}, J_{\theta Q_{e}}, J_{V P_{e}}, J_{V Q_{e}}, J_{\theta E_{q}^{\prime}}, J_{\theta E_{d}^{\prime}}, J_{V E_{q}^{\prime}} \text {, and } J_{V E_{d}^{\prime}}\right)^{2}$ represent the value of the Jacobian of $\Delta \theta$ and $\Delta V$ around the equilibrium point.

The state space system for the linearized two-axis model can be formulated by using (18) in (15), linearizing (2) and adding (18), and (19) in order to have:

$$
\left[\begin{array}{c}
\dot{\Delta} \delta \\
\dot{\Delta \omega} \\
\Delta \dot{E_{q}^{\prime}} \\
\Delta \dot{E}_{d}^{\prime}
\end{array}\right]=\left[\begin{array}{cccc}
0 & 1 & J_{\dot{\delta} E_{q}^{\prime}} & J_{\dot{\delta} E_{d}^{\prime}} \\
0 & 0 & 0 & 0 \\
0 & 0 & J_{\dot{E}_{q}^{\prime} E_{q}^{\prime}} & J_{\dot{E}_{q}^{\prime} E_{d}^{\prime}} \\
0 & 0 & J_{\dot{E}_{d}^{\prime} E_{q}^{\prime}} & J_{\dot{E}_{d}^{\prime} E_{d}^{\prime}}
\end{array}\right]\left[\begin{array}{c}
\Delta \delta \\
\Delta \omega \\
\Delta E_{q}^{\prime} \\
\Delta E_{d}^{\prime}
\end{array}\right]+
$$

\footnotetext{
${ }^{1}$ The appendix shows the detailed term

${ }^{2}$ detailed expressions are shown in the appendix
} 


$$
\begin{gathered}
{\left[\begin{array}{cc}
J_{\dot{\delta} P_{e}} & J_{\dot{\delta} Q_{e}} \\
-\omega_{0} / 2 H & 0 \\
J_{\dot{E}_{q}^{\prime} P_{e}} & J_{\dot{E}_{q}^{\prime} Q_{e}} \\
J_{\dot{E}_{d}^{\prime} P_{e}} & J_{\dot{E}_{d}^{\prime} Q_{e}}
\end{array}\right]\left[\begin{array}{c}
\Delta P_{e} \\
\Delta Q_{e}
\end{array}\right]+\left[\begin{array}{c}
J_{\dot{\delta} E_{f d}} \\
0 \\
1 / T_{d o}^{\prime} \\
0
\end{array}\right] \Delta E_{f d}} \\
{\left[\begin{array}{c}
\Delta \theta \\
\Delta V
\end{array}\right]=\left[\begin{array}{llll}
1 & 0 & J_{\theta E_{q}^{\prime}} & J_{\theta E_{d}^{\prime}} \\
0 & 0 & J_{V E_{q}^{\prime}} & J_{V E_{d}^{\prime}}
\end{array}\right]\left[\begin{array}{c}
\Delta \delta \\
\Delta \omega \\
\Delta E_{q}^{\prime} \\
\Delta E_{d}^{\prime}
\end{array}\right]+} \\
{\left[\begin{array}{ll}
J_{\theta P_{e}} & J_{\theta Q_{e}} \\
J_{V P} & J_{V Q_{e}}
\end{array}\right]\left[\begin{array}{c}
\Delta P_{e} \\
\Delta Q_{e}
\end{array}\right]}
\end{gathered}
$$

$J_{\dot{\delta} P_{e}}{ }^{2}, J_{\dot{\delta} Q_{e}}{ }^{2}$, and ${J_{\dot{\delta} E_{f d}}}{ }^{2}$ represent the influence of the input and the electric states $E_{q}^{\prime}$ and $E_{d}^{\prime}$ on the electromechanical state $\delta$.

The state space system (20)-(21) can be downsized into a system with two states only $\delta$ and $\omega$ with an additional error $\operatorname{Err} 1$ and $\operatorname{Err} 2$, since the equations of these two states include all the parameters in $\mathbf{M}$.

$$
\begin{aligned}
& {\left[\begin{array}{c}
\dot{\Delta} \delta \\
\dot{\Delta} \omega
\end{array}\right]=\left[\begin{array}{cc}
0 & 1 \\
0 & 0
\end{array}\right]\left[\begin{array}{c}
\Delta \delta \\
\Delta \omega
\end{array}\right]+\left[\begin{array}{cc}
J_{\dot{\delta} P_{e}} & J_{\dot{\delta} Q_{e}} \\
-\omega_{0} / 2 H & 0
\end{array}\right]\left[\begin{array}{c}
\Delta P_{e} \\
\Delta Q_{e}
\end{array}\right]+\operatorname{Err} 1} \\
& {\left[\begin{array}{c}
\Delta \theta \\
\Delta V
\end{array}\right]=\left[\begin{array}{cc}
1 & 0 \\
0 & 0
\end{array}\right]\left[\begin{array}{c}
\Delta \delta \\
\Delta \omega
\end{array}\right]+\left[\begin{array}{cc}
J_{\theta P_{e}} & J_{\theta Q_{e}} \\
J_{V P} & J_{V Q_{e}}
\end{array}\right]\left[\begin{array}{c}
\Delta P_{e} \\
\Delta Q_{e}
\end{array}\right]+E r r 2}
\end{aligned}
$$

In the above state space model, $J_{\dot{\delta} P_{e}}$ and $J_{\dot{\delta} Q_{e}}$ are determined by steady-state values of $E_{d}^{\prime}, E_{q}^{\prime}$, terminal voltage, rotor angle and time constants. These two terms, $J_{\text {deita } P_{e}}$ and $J_{\text {deita } Q_{e}}$, will be treated as independent parameters and will be estimated. $J_{\theta P_{e}}, J_{\theta Q_{e}}, J_{V P}$ and $J_{V Q_{e}}$ are all related to the transient reactance $x_{d}^{\prime}$. Hence these four terms are not independent parameters and will be expressed in terms of $x_{d}^{\prime}$.

In the case of a classical generator model, the dynamics of $E_{q}^{\prime}$ and $E_{d}^{\prime}$ are completely ignored, then $J_{\dot{\delta} P_{e}}$ and $J_{\dot{\delta} Q_{e}}$ are zeros.

As a summary, the system identification will identify these parameters for the proposed model: $H, x_{d}^{\prime}, J_{\dot{\delta} P_{e}}$ and $J_{\dot{\delta} Q_{e}}$, while identifying $H, x_{d}^{\prime}$ for the classical model.

The above system can be written as:

$$
\begin{aligned}
\dot{X} & =[A] X+[B] U+E r r 1 \\
Y & =[C] X+[D] U+E r r 2
\end{aligned}
$$

with the state vector $X=[\Delta \delta \Delta \omega]^{T}$, the observation (or measurement) vector $Y=[\Delta \theta \Delta V]^{T}$, the input vector $U=$ $\left[\Delta P_{e} \Delta Q_{e}\right]^{T}$, and the error vectors $\operatorname{Err} 1$ and $\operatorname{Err} 2$.

Note that the system matrix $[\mathrm{A}]$ is not stable. In differential equation integration, this can cause numerical error propagation. Therefore, this matrix is modified with small numerical number $\epsilon(\epsilon<0)$.

$$
A=\left[\begin{array}{ll}
0 & 1 \\
\epsilon & \epsilon
\end{array}\right]
$$

\section{Simulation AND VAlidation}

Matlab system identification toolbox is used for the case studies.

A four-machine two-area system is used for case study. Four sets of measurements for $P_{e}, Q_{e}, V$, and $\theta$ are taken following a three phase line to ground fault at a point right outside the machine or the power subsystem.

Small signal quantities $\Delta P_{e}, \Delta Q_{e}, \Delta V$ and $\Delta \theta$ are derived by removing the steady state value of the measurements. $\Delta P_{e}$ and $\Delta Q_{e}$ are considered as the input and $\Delta V$ and $\Delta \theta$ are considered as output. Both systems representing the proposed model and the classical model are implemented with MATLAB System Identification Toolbox grey box. For simplification and generalization purposes, $\operatorname{Err} 1$ and $\operatorname{Err} 2$ are treated as zeros.The simulation data is shown in the appendix.

Once the models are identified, the models of Fig. 3 are implemented in MATLAB Simulink and fed with the input $\Delta P_{e}, \Delta Q_{e}$ in addition to the estimated parameters. The output of the Simulink system and the states $(\Delta \delta$ and $\Delta \omega)$ are compared to the simulated output and mechanical states $\Delta \delta^{\prime}$ and $\Delta \omega$.

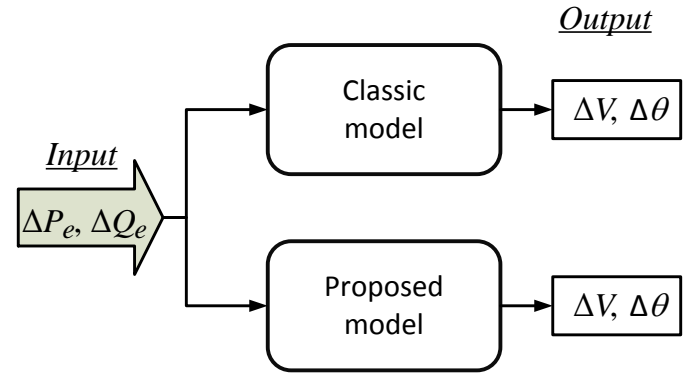

Fig. 3. Validation process of proposed model

The purpose of this case is to represent Area 1 machines (Fig. 4) by one single machine and run the estimation algorithm to find the equivalent machine parameters. Validation of the estimated machine will show if the equivalent machine truly represents Area 1. A similar approach can be used to represent Area 2 then the whole system can be scaled down to two equivalent machines connected by a radial transmission line.

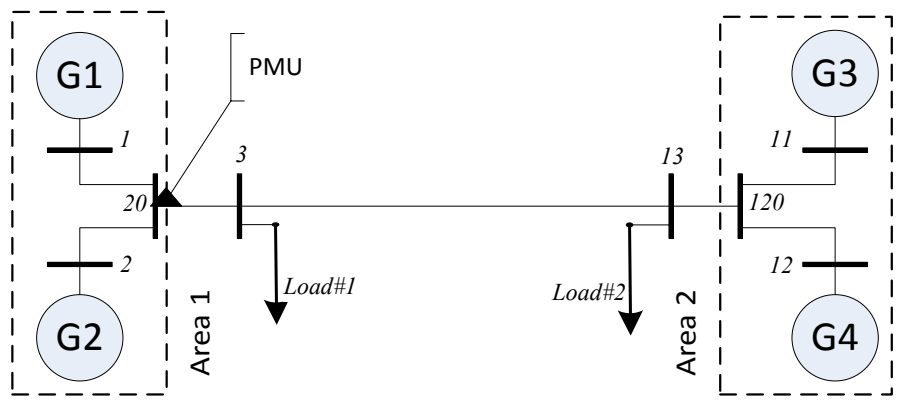

Fig. 4. Case 2: Four-machine two-Area System

The simulation was carried out in Power System Toolbox (PST) [22]. The simulated machines are similar and were built around sub-transient model and equipped with dc exciters and 


\begin{tabular}{|l|c|c|r|}
\hline Parameter & $H$ & $x_{d}^{\prime}$ & cost function \\
\hline Simulated equivalent machine & 13 & 0.27 & 0 \\
\hline Proposed model & 12.3 & 0.31 & $2.5 e_{-11}$ \\
\hline Classic model & 16 & 0.31 & $4.4 e_{-10}$ \\
\hline
\end{tabular}

CASE 2: ESTIMATED PARAMETERS OF THE PROPOSED MODEL AND CLASSIC MODEL

governors. The simulation details are shown in the appendix. Input and Output data were extracted in bus 20 were a PMU is supposed to be installed.

The resulting input and output data are shown in Fig. 5.

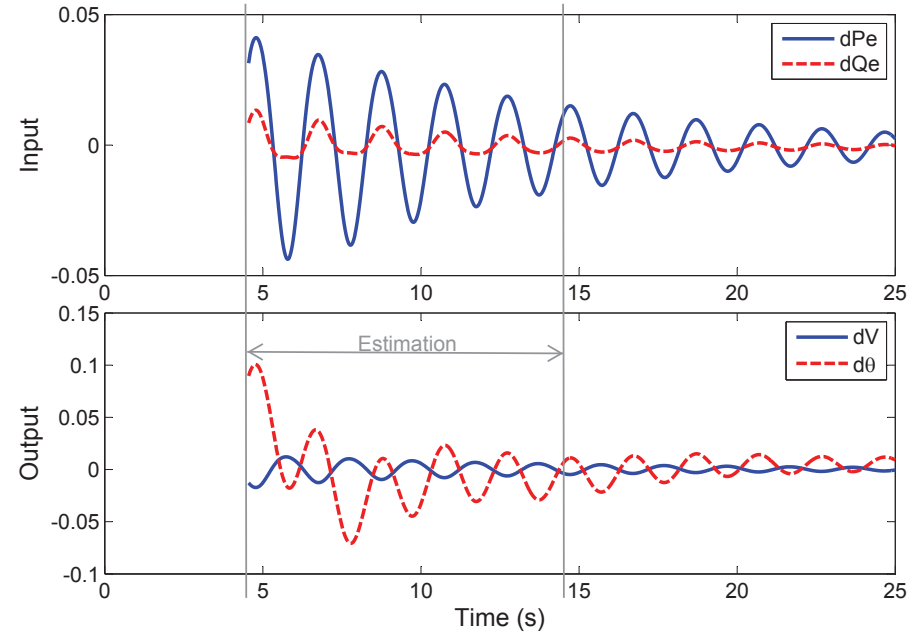

Fig. 5. Case 2: Input and Output

The stabilizing term $\epsilon$ formulated in (25) is set to -0.9 which is higher than -0.5 used in case 1 (subsection ??). The value -0.9 for $\epsilon$ is still small compared to the other factor $\left(-\omega_{0} / 2 \mathrm{H}\right)$ affecting $\Delta \dot{\omega}$ which is around -15 .

The estimated parameters along with the cost function ${ }^{3}$ provided by the system identification algorithm are shown in Table I. The simulated equivalent machine of the power subsystem has theoretically a total inertia equals the sum of the inertias of its individual machines (when perfectly coherent) and a transient reactance equals the Thevenin equivalent of the reactances seen from bus 20 (i.e. $H=13$ and $x_{d}^{\prime}=0.27$ ).

The validated output of the proposed model and the classic model are shown in 6. The impact of various values for $\epsilon$ on angle validation is shown in Fig. 7, which clearly shows the the proposed model is better in every case. The impact on $\Delta V$ was insignificant.

\section{CONCLusion}

This paper investigates using least square estimation based system identification to apply PMU data for generator model identification. A second-order model is proposed in this paper based the two-axis generator model. This model has inputs from real power and reactive power and outputs as the terminal voltage phasor. Unlike the classic generator model where the

\footnotetext{
${ }^{3}$ It is assumed that the simulated machine, being the base model, has a cost of 0
}
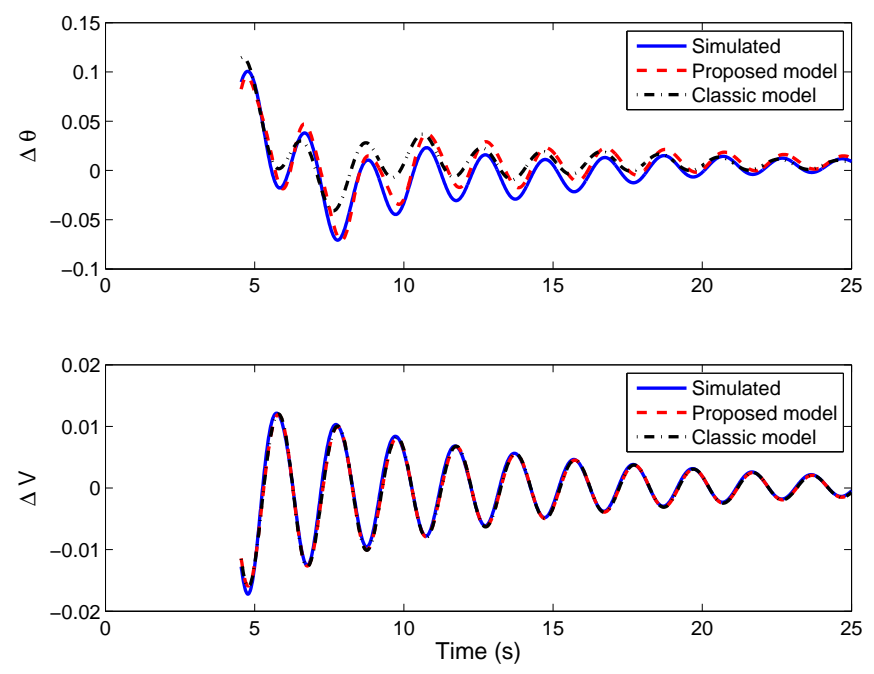

Fig. 6. Case 2: Validated output of the proposed model and classic model
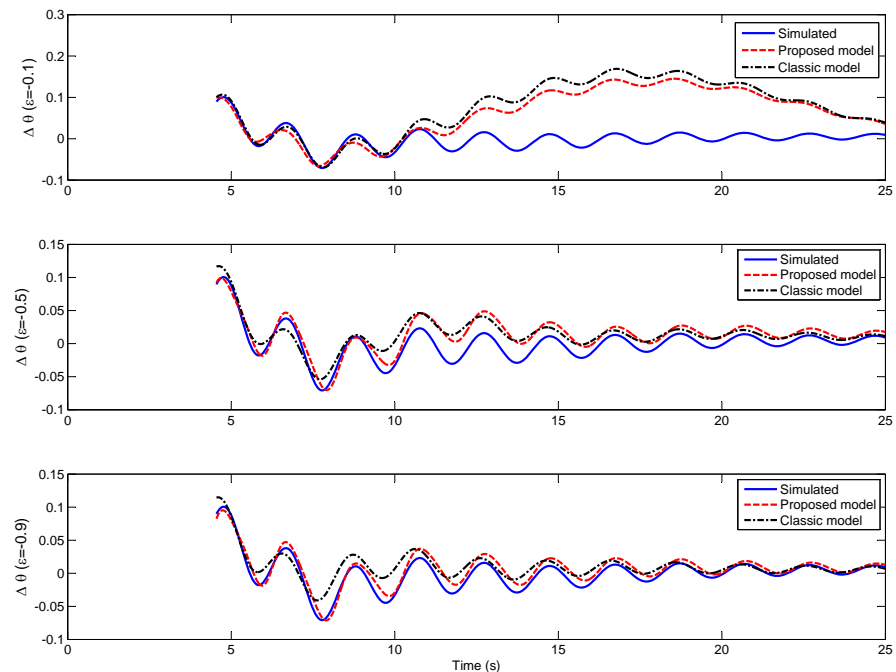

Fig. 7. Case 2: The impact of varying $\epsilon$ on the angle output of the proposed and classic models

dynamics of electric variables such as the internal voltages are completely ignored, this model includes the impact of these dynamics. System identification based on this model is demonstrated in two case studies: a single generator model identification and a subsystem identification. In both case studies, the proposed model is tested against the classical model and shows more accurate prediction of parameters and system responses.

\section{APPENDIX}

The detailed derivation of the Jacobian matrix is shown in the longer version of the paper posted at http://power.eng.usf.edu.

Case 1: Simulation data for the machine: $H=$ $3.7 \mathrm{~s}, x_{d}^{\prime}=0.4 \mathrm{pu}, x_{q}^{\prime}=0.4 \mathrm{pu}, x_{d}=1.81 \mathrm{pu}, x_{q}=$ $1.81 \mathrm{pu}, r_{s}=0, x_{d}^{\prime \prime}=0.15 \mathrm{pu}, x_{q}^{\prime \prime}=0.15 \mathrm{pu}, T_{d 0}^{\prime}=$ $8 s, T_{q 0}^{\prime}=1 s, T_{d 0}^{\prime \prime}=0.03, T_{q 0}^{\prime \prime}=0.07 \mathrm{~s}$. Exciter: $T_{r}=0 \mathrm{~s}, K_{a}=200, T_{a}=0.03 \mathrm{~s}, K_{e}=1, T_{e}=$ 
$0.1 s, T_{b}=5 s, T_{c}=0.3 s, K_{f}=2.5 e^{-3}, T_{f}=$ $0.21 s, E f_{\min }=-11.5, E f_{\max }=11.5, K_{p}=0$

Case 2:

Simulation data for the machines: $H=6.5 s, x_{d}^{\prime}=$ $0.3 p u, \quad x_{q}^{\prime}=0.3 p u, \quad x_{d}=1.81=p u, x_{q}=$ $1.7 \mathrm{pu}, r_{s}=0.0025, x_{d}^{\prime \prime}=0.25 \mathrm{pu}, x_{q}^{\prime \prime}=0.25 \mathrm{pu}, T_{d 0}^{\prime}=$ $8 s, T_{q 0}^{\prime}=0.4 s, T_{d 0}^{\prime \prime}=0.03, T_{q 0}^{\prime \prime}=0.05 s$. DC Exciter: $T_{r}=0.01 s, K_{a}=46, T_{a}=0.06 \mathrm{~s}, K_{e}=0, T_{e}=$ $0.46 s, T_{b}=0 s, T_{c}=0 s, K_{f}=0.1, T_{f}=1 s, V r_{\min }=$ $-0.9, V r_{\max }=1$

\section{REFERENCES}

[1] A. Phadke and R. de Moraes, "The wide world of wide-area measurement," Power and Energy Magazine, IEEE, vol. 6, no. 5, pp. 52 -65, september-october 2008.

[2] Z. Huang, P. Du, D. Kosterev, and B. Yang, "Application of extended kalman filter techniques for dynamic model parameter calibration," in Power Energy Society General Meeting, 2009. PES '09. IEEE, july 2009, pp. $1-8$.

[3] K. Kalsi, Y. Sun, Z. Huang, P. Du, R. Diao, K. Anderson, Y. Li, and B. Lee, "Calibrating multi-machine power system parameters with the extended kalman filter," in Power and Energy Society General Meeting, 2011 IEEE, july 2011.

[4] L. Fan and Y. Wehbe, "Extended kalman filtering based real-time dynamic state and parameter estimation using pmu data," Electric Power Systems Research, vol. 103, pp. 168-177, 2013.

[5] Y. Wehbe and L. Fan, "UKF based estimation of synchronous generator electromechanical parameters from phasor measurements," in North American Power Symposium (NAPS), 2012, sep. 2012.

[6] J.-Y. Choi, B. Cho, H. VanLandingham, H. soo Mok, and J.-H. Song, "System identification of power converters based on a black-box approach," Circuits and Systems I: Fundamental Theory and Applications, IEEE Transactions on, vol. 45, no. 11, pp. 1148 -1158, nov 1998.

[7] B. Miao, R. Zane, and D. Maksimovic, "System identification of power converters with digital control through cross-correlation methods," Power Electronics, IEEE Transactions on, vol. 20, no. 5, pp. 1093 1099, sept. 2005.

[8] K. Chau and C. Chan, "Nonlinear identification of power electronic systems," in Power Electronics and Drive Systems, 1995., Proceedings of 1995 International Conference on, feb 1995, pp. 329 -334 vol.1.

[9] L. Xu and L. Fan, "System identification based vsc-hvdc dc voltage controller design," in North American Power Symposium (NAPS), 2012, sep. 2012.

[10] L. Fan, Z. Miao, and Y. Wehbe, "Application of dynamic state and parameter estimation techniques on real-world data," IEEE Transactions on Smart Grid, vol. 4, no. 2, pp. 1133-1141, 2013.

[11] J. Pierre, N. Zhou, F. Tuffner, J. Hauer, D. Trudnowski, and W. Mittelstadt, "Probing signal design for power system identification," Power Systems, IEEE Transactions on, vol. 25, no. 2, pp. 835 -843, may 2010.

[12] J. Khazaei, L. Fan, W. Jiang, and D. Manjure, "Distributed prony analysis on real-world pmu data," submitted, IEEE Trans. Power Systems, 2015.

[13] B. Mogharbel, L. Fan, and Z. Miao, "Least squares estimation-based synchronous generator parameter estimation using pmu data," accepted, IEEE PESGM 2015, 2015.

[14] L. Fan, "Least squares estimation and kalman filter based dynamic state and parameter estimation," accepted, IEEE PESGM 2015, 2015.

[15] I. Kamwa and L. Gerin-Lajoie, "State-space system identification-toward mimo models for modal analysis and optimization of bulk power systems," Power Systems, IEEE Transactions on, vol. 15, no. 1, pp. $326-335$, feb 2000.

[16] M. Burth, G. Verghese, and M. Velez-Reyes, "Subset selection for improved parameter estimation in on-line identification of a synchronous generator,' Power Systems, IEEE Transactions on, vol. 14, no. 1, pp. 218 -225 , feb 1999.

[17] A. Cintron-Arias, H. T. Banks, A. Capaldi, and A. L. Lloyd, "A sensitivity matrix based methodology for inverse problem formulation." Journal of Inverse \& Ill-Posed Problems, vol. 17, no. 6, pp. 545 - 564, 2009.

[18] P. Sauer and M. Pai, Power System Dynamics and Stability. Prentice Hall, 1998.
[19] J. Machowski, Power system dynamics stability and control. Chichester, U.K: Wiley, 2008.

[20] Y. Bard, Nonlinear parameter estimation. New York: Academic Press, 1974.

[21] J. R. S. H. Thomas Banks, Marie Davidian and K. L. Sutton, MATHEMATICAL AND STATISTICAL ESTIMATION APPROACHES IN EPIDEMIOLOGY. Springer, 2009, ch. An Inverse Problem Statistical Methodology Summary, pp. 249-302.

[22] J. Chow, G. Rogers, and K. Cheung, "Power System Toolbox," Tech. Rep. 